\title{
Delayed radiation therapy timing and use of intensity-modulated radiation therapy in non-head and neck pediatric rhabdomyosarcoma
}

\author{
Bree R. Eaton • Howard M. Katzenstein • \\ Anna L. Sutter • Mourad Tighiouart • \\ Karen Wasilewski-Masker • Muna Qayed • \\ Bradley George • Thomas A. Olson • Natia Esiashvili
}

Received: 25 May 2012 / Accepted: 2 July 2012 /Published online: 20 July 2012

(C) Springer-Verlag 2012

\begin{abstract}
Objective The impact of radiation therapy (RT) timing and use of intensity-modulated radiation therapy (IMRT) in nonhead and neck pediatric rhabdomyosarcoma (RMS) is underreported.

Methods Children with non-head and neck RMS treated definitively with chemotherapy and RT from December 2000 through May 2010 at our institution were identified for analysis. Kaplan-Meier estimates of time to local progression-free survival (PFS), disease-free survival, and overall survival were performed, and Cox proportional hazard model examined the relationship between local control and RT timing and the use of IMRT.
\end{abstract}

B. R. Eaton $(\bowtie) \cdot$ A. L. Sutter $\cdot$ N. Esiashvili

Department of Radiation Oncology, Winship Cancer Institute,

Emory University,

Building A, Suite CT 104,

Atlanta, GA 30322, USA

e-mail: brupper@emory.edu

H. M. Katzenstein · K. Wasilewski-Masker • M. Qayed •

B. George $\cdot$ T. A. Olson

Department of Pediatric Hematology-Oncology,

Aflac Cancer Center, Children's Healthcare of Atlanta,

Atlanta, GA, USA

H. M. Katzenstein · K. Wasilewski-Masker • M. Qayed •

B. George $\cdot$ T. A. Olson

Children's Healthcare of Atlanta, Emory University,

Atlanta, GA, USA

M. Tighiouart

Biostatistics and Biomedical Informatics, Samuel Oschin

Comprehensive Cancer Insitute,

Los Angeles, CA, USA
Results Thirty seven children, including 25 patients with non-metastatic disease, 12 patients with metastatic disease, and a total of 44 tumors were identified. Three-year local PFS was $87 \%$ (95 \% CI: $0.79-0.93)$ for the non-metastatic group and $76 \%(95 \% \mathrm{CI}: 0.63-0.88)$ for the metastatic group. Among the non-metastatic group, the median time from the start of chemotherapy to RT was 17 weeks (range 4-43) with only four patients treated within $\leq 12$ weeks. Median RT timing for the metastatic group was 21 weeks (range 15-68). Among all patients, IMRT was used in 17 (39\%) cases. No significant association between local control and RT timing or use of IMRT was found.

Conclusion Our data demonstrate good rates of local tumor control with delayed RT timing of greater than 12 weeks and the use of IMRT in a subset of pediatric patients with nonhead and neck RMS.

Keywords Pediatric hematology/oncology ·

Rhabdomyosarcoma $\cdot$ Radiation therapy $\cdot$ IMRT

\section{Introduction}

Rhabdomyosarcoma (RMS) is the most common soft tissue sarcoma of childhood, yet remains a rare disease with approximately 350 new cases diagnosed annually in USA [1]. This patient population is highly varied as RMS can involve any soft tissue site within the body and includes multiple histological subtypes. From 1972 to 1997, the Intergroup Rhabdomyosarcoma Study Group (IRSG) led five multi-institutional randomized trials investigating the effect of multiple patient and tumor characteristics and treatment variables on patient outcomes. Results have guided national standards for risk stratification 
and the use of multi-modality therapy. For all patients with alveolar histology (ARMS) or microscopic or gross residual disease, radiation therapy (RT) has been a standard component of definitive treatment as a review of IRSG studies I-III demonstrated a $25-30 \%$ improvement in failure-free survival at 10 years for these patients [1,2]. Patients with metastases have also been shown to benefit from local therapy and typically receive RT in definitive doses to the primary tumor as well as to sites of metastases. Standard RT for RMS is delivered in oncedaily fractions to doses between 36 and $54 \mathrm{~Gy}$, varying according to group, histologic subtype, and status of regional lymph nodes and/or distant metastases [1-4].

While the benefit of RT is widely accepted, no level 1 data or consensus guidelines exist regarding specific radiation therapy parameters such as RT timing or the use of intensity-modulated radiation therapy (IMRT). To date, published literature evaluating the impact of these variables is limited to patients with parameningeal (PM) RMS [5-8]. In a review of patients with PM RMS treated on IRSG studies II-IV, early initiation of RT within 2 weeks of diagnosis improved local control at 5 years for patients with evidence of meningeal impingement or intracranial extension [5], and this analysis has supported the early initiation of RT for this subgroup in subsequent cooperative group protocols. Due to the sensitive location of parameningeal primary tumors, IMRT is often employed in attempt to reduce the RT dose to critical structures and our own institutional experience with this technique, among others, has previously been reported [6]. However, the effect of RT timing or use of IMRT on disease outcomes has not yet been evaluated in patients with RMS of non-head and neck primary sites. Herein, we examine the relationship between local control and RT timing, and the use of IMRT in children with RMS of the trunk and extremity who were treated at our institution.

\section{Methods}

\section{Patient population}

The treatment records of the Emory Clinic and Children's Healthcare of Atlanta were evaluated to identify all patients treated with RT for RMS between December 2000 and May 2010. Outcome of patients with primary head and neck parameningeal sites were previously reported from our institution [6] and were excluded from this analysis. The current study cohort includes all non-head and neck disease sites treated in the selected time period. Institutional review board approval was obtained for this retrospective analysis.

All the patients had pathological confirmation of RMS diagnosis based on biopsy or surgical resection. The staging evaluation included computed tomography (CT) and/or magnetic resonance imaging (MRI) of the primary site, and fludeoxyglucose positron emission tomography (FDG-PET) in two cases. A
$\mathrm{CT}$ of the chest, a technetium ${ }^{99}$ bone scan, and bone marrow aspirate and biopsy were performed on all patients to evaluate for distant metastasis. Cases were assigned both a pretreatment stage according to the International Union against Cancer staging system used by the IRSG [9] and a postoperative group according to the IRSG system [10]. Systemic chemotherapy was administered after pathologic confirmation of diagnosis and completion of systemic work-up and staging. Radiation therapy was initiated at a time period according to chemotherapy protocol or varied based on patient referral, delays in chemotherapy, or other social or patient-specific factors.

\section{Radiation therapy}

CT simulation with immobilization specific to disease site was used for treatment planning. General anesthesia was given for simulation and daily treatment when necessary for immobilization. The pre- and post-chemotherapy MRI, FDG-PET (when available), and CT scans were registered to the simulation $\mathrm{CT}$ for delineation of the target volume. The gross tumor volume (GTV) was defined as the extent of gross soft tissue abnormality on contrast-enhancing CT or MRI. Both pre- and post-chemotherapy GTVs were created when applicable. Pre- and post-chemotherapy clinical tumor volumes (CTV) were created by expansion of the corresponding GTV with 0.5 - to $1.5-\mathrm{cm}$ margin to account for potential microscopic tumor extension. The margins were modified according to the anatomic location of the tumor to respect natural barriers to tumor spread such as bone. An initial and boost PTV were created to account for patient setup uncertainty and target motion by the addition of a $0.5-$ to $1-\mathrm{cm}$ margin to the pre-chemotherapy and postchemotherapy CTV, respectively.

RT dose was specified according to pretreatment stage and postoperative group and ranged from 36-54 Gy. In the case of field reduction for PTV boost, boost was initiated between 36 and 41.4 Gy. RT delivery included external beam radiation therapy (EBRT) photons of $6 \mathrm{MV}$ or higher energy using conventional field arrangements or IMRT. IMRT consisted of 6-7 beam field arrangements (Fig. 1). RT dose was prescribed such that the entire PTV should be encompassed within the $95 \%$ isodose surface and no more than $10 \%$ of the PTV should receive greater than $110 \%$ of the prescribed dose. Electron beam therapy and high-dose rate (HDR) brachytherapy were also used in selected cases. Contouring and treatment planning was done with Eclipse software (Varian Medical Systems, Palo Alto, CA). Onboard kV imaging was used to ensure setup accuracy for treatment delivery when available.

Statistical analysis

Local progression-free survival (PFS), disease-free survival (DFS), and overall survival (OS) were estimated using the 
Fig. 1 IMRT plans including a seven-field arrangement to the abdomen (a) and a six-field arrangement to the prostate (b). The resulting high-dose region (red corresponds to $100 \%$ of prescribed dose) allowing for comprehensive coverage of the tumor volume in blue, while the sparing the liver and kidney (c) and the bladder and rectum (d). The low-dose region in $\mathbf{d}$ (orange corresponds to $15 \%$ of the prescribed dose) also illustrates sparing of the femoral heads

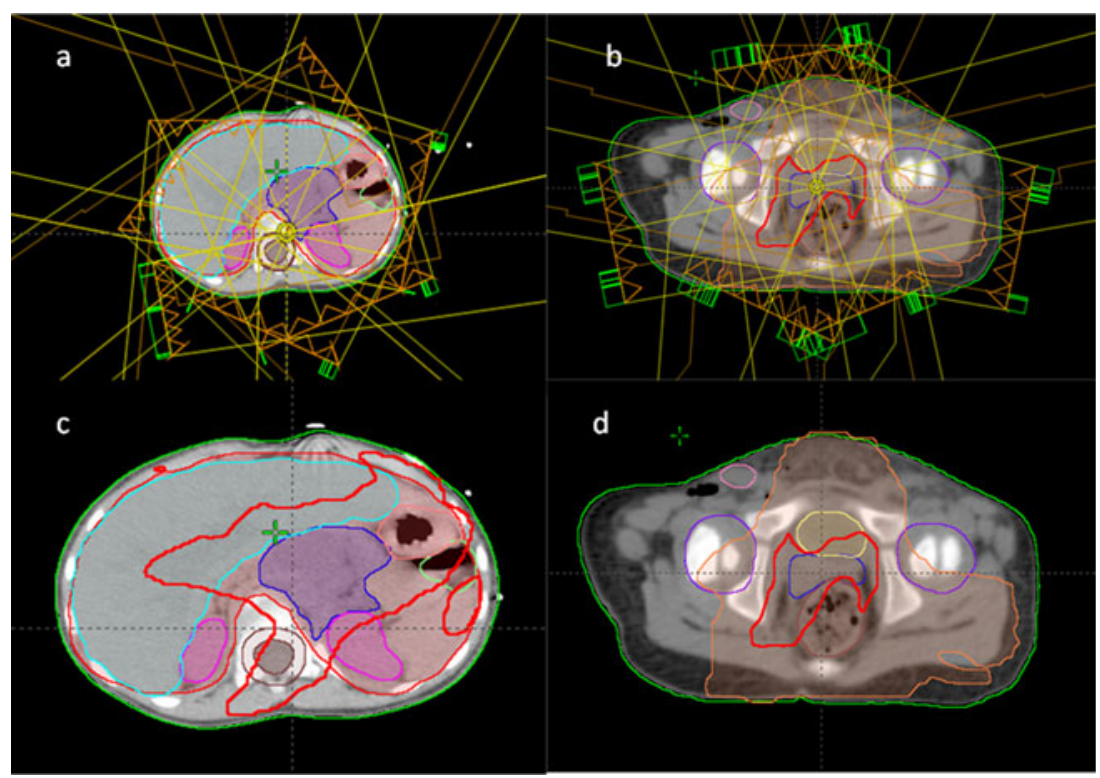

Kaplan-Meier method. Cox proportional hazard model examined the relationship between local control and RT timing, use of IMRT, histology, presence of metastatic disease, disease group, and stage. SPSS statistics version 19 was used to carry out statistical analysis.

Local failure was defined as tumor recurrence or progression at the primary site as evident on radiographic imaging or physical exam and was calculated for each tumor included for analysis. Local PFS was calculated from the time of chemotherapy initiation until first evidence of local failure. DFS was defined as being alive with no evidence of local or distant relapse of RMS. OS survival was calculated from the start of chemotherapy until time of death or was censored at last follow-up. RT timing was defined as the time from chemotherapy initiation to the time of RT initiation. For patients with metastatic disease, RT timing was separately determined for each treated tumor site.

\section{Results}

\section{Patient characteristics}

A total of 37 children with non-head and neck RMS, including 25 patients (25 tumors) with non-metastatic disease and 12 patients (19 tumors) with metastatic disease treated with definitive RT upon initial diagnosis were evaluated in the present study. Patient and tumor characteristics are detailed in Table 1.

\section{Chemotherapy}

All but two patients (95\%) received multi-agent chemotherapy according to specified cooperative group protocol, either on or off study. Chemotherapy regimens included Children's Oncology Group (COG) intermediate-risk protocol D9803 using vincristine, dactinomycin, and cyclophosphamide (VAC) or VAC alternating with vincristine, topotecan, and cyclophosphamide (14 patients); the "Mayo regimen" using vincristine, doxorubicin, and cyclophosphamide, alternating with isophosphamide and etoposide (10 patients); ARST0331 consisting of vincristine and dactinomycin with low-dose cyclophosphamide (four patients); low-risk regimen of IRSG-V (COG D9602), using vincristine and dactinomycin (one patient), ARST0531with either VAC or VAC alternating with infusions of vincristine and irinotecan (four patients); and the high-risk regimen of ARST0431using vincristine, doxorubicin, and cyclophosphamide alternating with isophosphamide and etoposide (three patients). Chemotherapy regimens used unknown to be part of specified protocol included cytoxan/topotecan (one patient) and etoposide alone (one patient).

\section{Radiation therapy}

RT parameter data for both the non-metastatic and metastatic groups are summarized in Table 2.

\section{Non-metastatic group}

For patients without metastatic disease, the median time from the start of chemotherapy to RT was 17 weeks (range 4-43), with only four patients treated within $\leq 12$ weeks. All patients completed a single course of definitive RT to the primary tumor site. Twenty-two patients $(88 \%)$ in the nonmetastatic group were treated with EBRT and of these, 10 (40 \%) received IMRT. One patient received EBRT followed by vaginal cylinder HDR brachytherapy and three 
Table 1 Patient and disease characteristics

\begin{tabular}{|c|c|c|}
\hline Characteristic & Non-Metastatic $n=25$ & Metastatic $n=12$ \\
\hline \multicolumn{3}{|l|}{ Gender } \\
\hline Male & 12 & 6 \\
\hline Female & 13 & 6 \\
\hline \multicolumn{3}{|l|}{ Age } \\
\hline Median & 3 & 5 \\
\hline Range & $1-15$ & $3-17$ \\
\hline \multicolumn{3}{|l|}{ Primary Site } \\
\hline Extremity & 6 & 3 \\
\hline Bladder/Prostate & 5 & 2 \\
\hline GU, non-bladder/non-prostate & 5 & 2 \\
\hline Trunk, rertoperitoneum, ect. & 9 & 5 \\
\hline \multicolumn{3}{|l|}{ Histologic Type } \\
\hline Embryonal & 16 & 4 \\
\hline Alveolar & 9 & 7 \\
\hline NOS & 0 & 1 \\
\hline \multicolumn{3}{|l|}{ IRSG Stage } \\
\hline I & 5 & \\
\hline II & 4 & \\
\hline III & 16 & \\
\hline IV & & 12 \\
\hline \multicolumn{3}{|l|}{ IRSG Group } \\
\hline II & 5 & \\
\hline III & 20 & \\
\hline IV & & 12 \\
\hline
\end{tabular}

patients received vaginal cylinder HDR alone. Median RT dose was 41.4 Gy (range 24-54 Gy). Daily fraction dose for patients receiving EBRT was $1.8 \mathrm{~Gy}$, except for two patients receiving whole abdomen RT (one patient at 1.25 Gy per fraction and one patient at $1 \mathrm{~Gy}$ per fraction delivered twice daily). All vaginal HDR treatments were delivered in 3 Gy per fraction, ranging from 6 to 12 fractions using Ir-192.

PTV was reduced for cone-down boost, based on response to induction chemotherapy, in nine patients $(36 \%)$ with a median relative reduction in boost PTV volume as compared with initial PTV volume of $45 \%$ (range 21$96 \%$ ). Eight of the nine patients (88\%) treated with PTV reduction had group 3 disease, and two (22\%) of these patients underwent resection of residual disease after completion of radiotherapy. Treatment to the reduced PTV boost volume began after median dose of 36 Gy (range 30.6-41.4 Gy).

\section{Metastatic group}

Among the 12 patients with metastatic disease, 19 tumors were treated with RT with a definitive intent for improved local control. Median RT timing and dose for this group was 21 weeks (range 15-68) and 50.4 Gy (range 36-50.4 Gy), respectively. RT timing for each of all RTs consisted of EBRT, and seven cases (37\%) utilized IMRT with the goal of reducing dose to abdominal organs at risk such as small bowel and kidney. Median total RT dose was 50.4 Gy (range 36-50.4 Gy).
Table 2 Radiation therapy parameters

\begin{tabular}{lll}
\hline & Non-Metastatic Group $n=25$ & Metastatic Group $n=19$ \\
\hline $\begin{array}{l}\text { RT Dose } \\
\text { Median (range) }\end{array}$ & 41.4 Gy (24-54) & 50.4 Gy (26-50.4) \\
RT timing & & \\
$\quad$ Median (range) & 17 weeks (4-43) & 21 weeks $(15-68)$ \\
$n(\%)>12$ weeks & $21(84)$ & $19(100)$ \\
IMRT & $10(40)$ & $7(37)$ \\
$n(\%)$ &
\end{tabular}


Table 3 Tumor and RT characteristics for observed local failures

\begin{tabular}{lllcccc}
\hline Stage, group & Histology & Location & RT Timing (weeks) & RT Dose (Gy) & IMRT & Time to failure (months) \\
\hline III, III & Alveolar & Foot & 14 & 50.4 & No & 16.3 \\
III, III & Embryonal & Prostate & 11 & 50.4 & Yes & 12.9 \\
III, III & Embryonal & Prostate & 13 & 50.4 & Yes & 12.8 \\
IV & Alveolar & Chest wall & 19 & 50.4 & No & 15.4 \\
IV & Alveolar & Mediastinum & 25 & 36 & No & 45.6 \\
IV & Embryonal & Abdomen & 15 & 50.4 & Yes & 24.8 \\
IV & Embryonal & Abdomen & 67 & 36 & Yes & 27.1 \\
\hline
\end{tabular}

PTV was reduced for boost, based on response to induction chemotherapy, in eight (38\%) RT courses with a median relative reduction in boost PTV volume as compared with initial PTV volume of $57 \%$ (range 27-97\%). One patient treated with PTV reduction underwent tumor resection following chemotherapy and prior to initiation of RT. PTV boost with reduced volume began after median dose of 36 Gy (range 24-41.4 Gy) delivered to the initial PTV.

\section{Patient Outcomes}

\section{Non-metastatic group}

At a median follow-up of 40 months (range 9-122), all patients without metastatic disease are alive, 20 patients ( $80 \%)$ have no evidence of disease, $3(12 \%)$ have stable residual disease after therapy, and two ( $8 \%$ ) have progressive disease. Three patients (12\%) experienced local failure, and tumor characteristics, treatment, and recurrence data for these three patients are detailed in Table 3. Kaplan-Meier estimates of 3-year local PFS and overall DFS are both $87 \%$ (95 \% CI: 0.79-0.93, Figs. 2 and 3). One regional and one distant failure occurred after 57 and 44 months, respectively. No significant association between local PFS and RT timing $(p=0.29)$ or use of IMRT $(p=0.29)$ was found.

\section{Metastatic group}

At a median follow-up of 29 months, eight patients (66\%) with metastatic disease are alive with a median survival of 40.1 months. Three patients $(25 \%)$ have no evidence of disease, seven $(41 \%)$ have persistent or progressive disease, and eight patients $(66 \%)$ have died. Kaplan-Meier estimate of 3-year OS is $61 \%(95 \%$ CI: $0.45-0.76$, Fig. 3). Among the metastatic group, 19 tumors present at initial diagnosis and treated with definitive RT were analyzed for local control. Results showed a 3-year local PFS of $70 \%$ (95 \% CI: $0.55-0.85$, Fig. 3). There were four (21\%) local failures for which RT was initiated at a median time of 22 weeks (range 15-68). Additionally, a

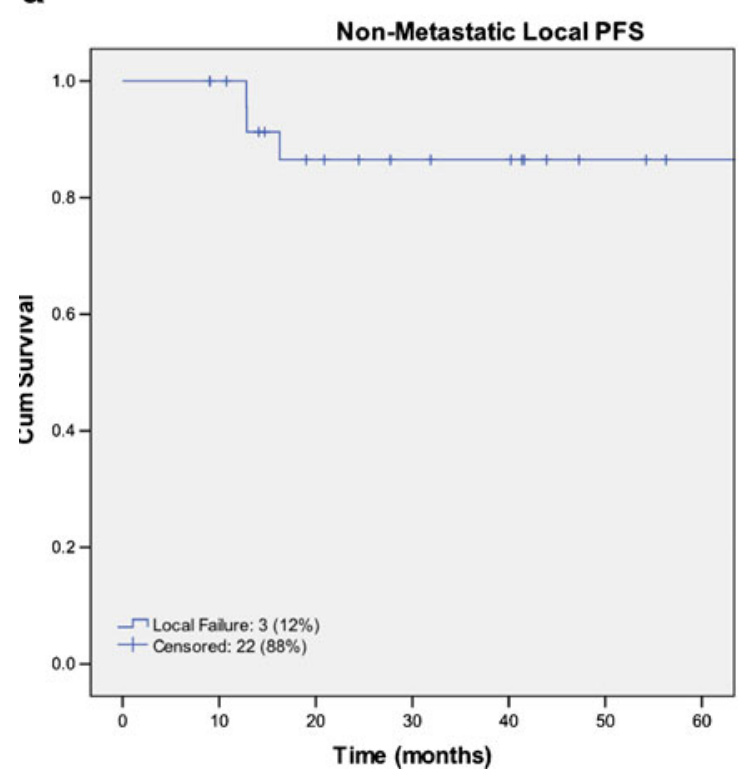

b

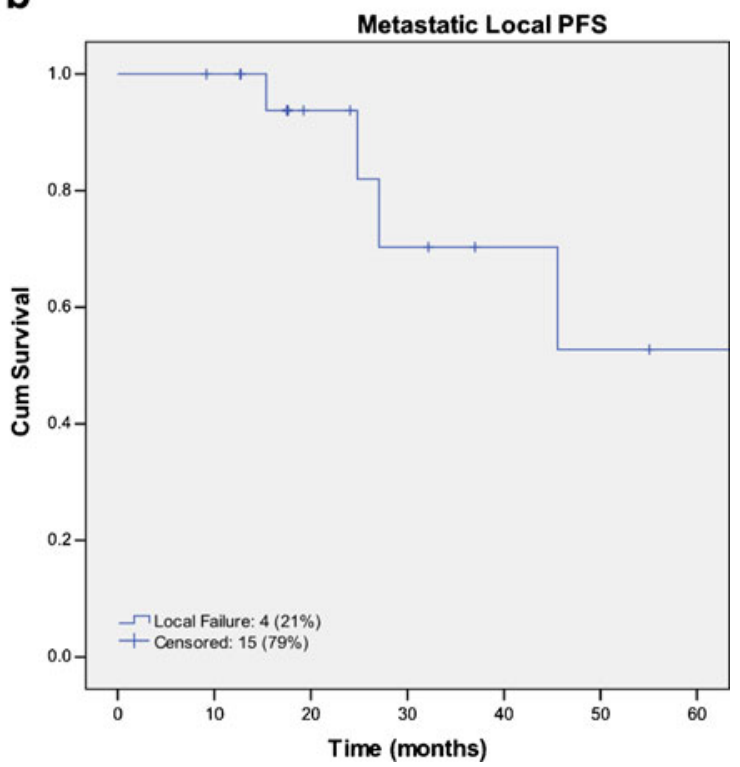

Fig. 2 Kaplan-Meier curves of local progression-free survival for patients without metastatic disease (a) and patients with metastatic disease (b) 

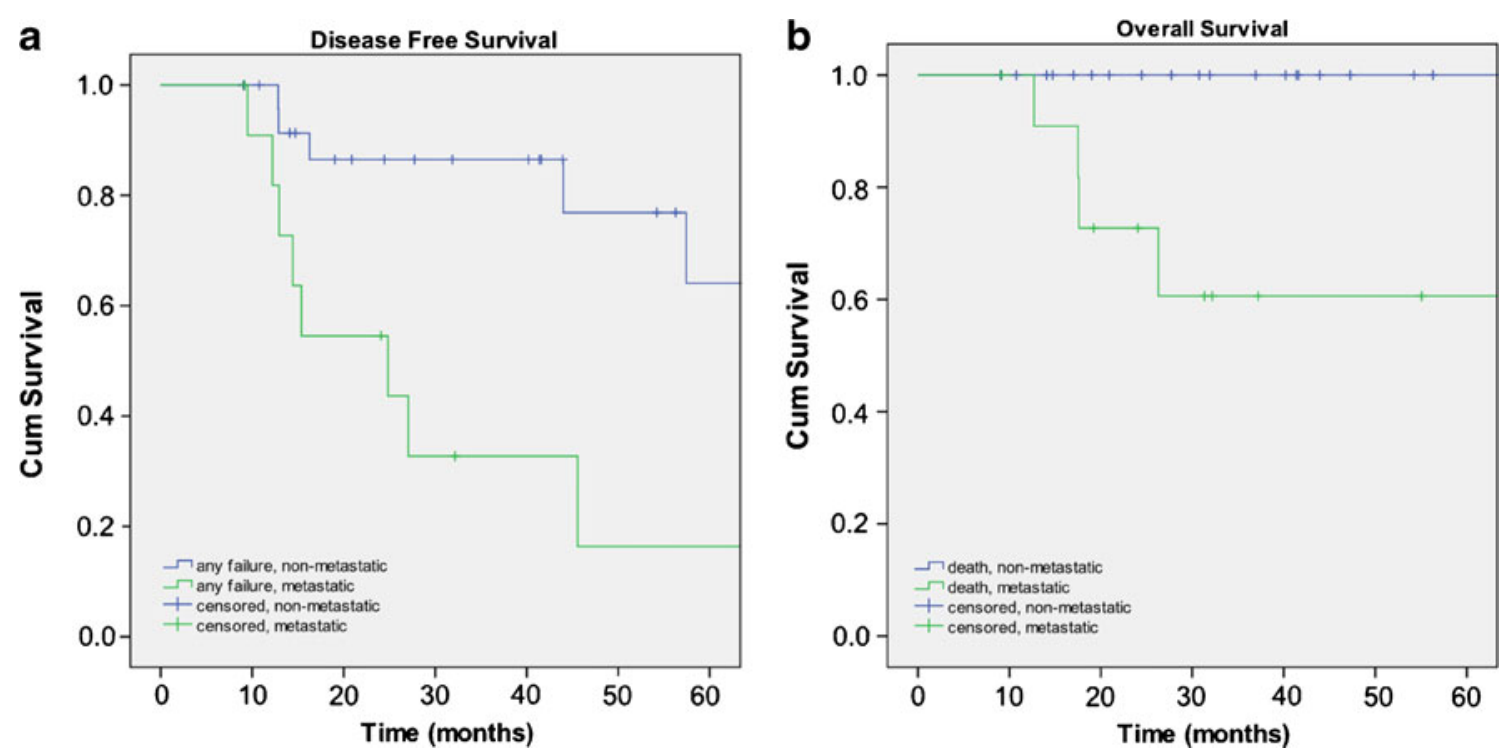

Fig. 3 Kaplan-Meier curves of disease-free survival (a) and overall survival (b) for patient with and without metastatic disease

no significant association between local PFS and RT timing $(p=0.37)$ or the use of IMRT $(p=0.94)$ was found among the metastatic group.

\section{Combined analysis of radiation therapy parameters}

In combined analysis of all 44 tumors treated, seven (16\%) local failures were observed. Similar to the subgroup analysis performed above, on combined univariate and multivariate analysis, no significant association was found between local control and RT timing or the use of IMRT.

\section{Discussion}

Our data demonstrate good rates of local control in a subset of pediatric patients with non-head and neck RMS, for which RT was initiated at a median time of 20 weeks after the start of chemotherapy, and IMRT was used in greater than one-third of the population. The 3-year local PFS of $87 \%$ observed in our analysis is comparable with historical controls as demonstrated by results of IRSG-IV, where 3year EFS was $77 \%$ and the local failure rates for non-head and neck sites varied from $7 \%$ for extremity to $14-19 \%$ for all other sites $[11,12]$. There was no recognizable detriment to tumor control with delayed RT timing or the use of IMRT during treatment. This is the first publication to specifically address the effect of these RT parameters on patient outcomes in pediatric RMS of non-head and neck primary sites.

Our results would suggest that delayed radiation therapy does not compromise local control in non-head and neck RMS as has been previously reported in parameningeal primary sites with evidence of intracranial extension of meningeal impingement [5]. The three local failures observed in the non-metastatic group occurred in patients with RT timing of 10,13 , and 14 weeks, actually representing relatively early RT timing in comparison with the median of 17 weeks for the entire non-metastatic group. However, only four patients $(16 \%)$ initiated RT at what would be considered early timing (i.e., $\leq 12$ weeks) and thus, our data are predominantly a review of late RT timing, and conclusions about early versus later RT timing in relationship to patient outcomes cannot be drawn. The three local failures all occurred in patients with multiple known poor prognostic factors including group 3 disease and unfavorable sites, suggesting that our observed local failure rate represents expected outcomes in comparison with historical controls, rather than a consequence of delayed RT timing or the use of IMRT.

Furthermore, delayed RT timing and the use of IMRT allowed for RT treatment volume to be reduced based on tumor response to induction chemotherapy and the use of reduced GTV to PTV expansion margins. Such a reduction in treatment volume could potentially be expected to reduce both acute and long term RT toxicity in this vulnerable pediatric population. While there is a concern for increased risk of secondary malignancies with the use of IMRT due to the stochastic effect of low dose radiation received to a larger treatment volume, it is important to point out that IMRT was used in prostate and abdomen cases where most often at least four radiation beams are used even with conventional planning. Thus, the six-field IMRT plans illustrated here would be expected to only minimally increase the integral RT dose as compared with conventional RT plans, and when combined with ability to significantly 
reduce RT treatment volume, it could be argued that IMRT may in fact present a reduced risk for such late effects.

In analysis of our findings, it is important to consider the inherent limitations of this retrospective review. We found no negative effect of delayed RT timing or the use of IMRT on local control, yet the small number of patients, heterogeneous population, and few local failure events available for analysis limit our ability to detect a statistically significant relationship. Although we comment on the expectation that smaller PTV volumes would lead to reduced toxicity, insufficient documentation prevented direct analysis of RT toxicity in this report. Still, our institutional review adds to the limited published data regarding radiation therapy specific parameters for RMS of non-head and neck sites and to our knowledge is the first report to describe and analyze the use of delayed RT and IMRT in this patient population. Further analysis is required to define the optimal radiation therapy timing and evaluate the direct effects of these RT variables on treatment toxicity.

\section{Conclusion}

Our institutional review suggests that neither delayed radiation therapy timing nor the use intensity-modulated radiation therapy compromises local tumor control among nonhead and neck primary pediatric rhabdomyosarcoma. Furthermore, delayed RT timing and the use of IMRT allowed for significant reductions in PTV volume for cone-down boost. These results should be validated in large prospective cohorts and further analysis is required to evaluate the potential for reduced treatment toxicity with PTV volume reduction.

Conflict of interest The authors state that they have no conflict of interest.

\section{References}

1. Punko JA, Mertens AC, Baker KS et al (2005) Long-term survival probabilities of childhood rhabdomyosarcoma: a population-based analysis. Cancer 103:1475-83

2. Wolden S, Anderson J, Crist W et al (1999) Indications for radiotherapy and chemotherapy after complete resection in rhabdomyosarcoma: a report from the intergroup rhabdomyosarcoma studies I-III. J Clin Oncol 17:3468-3475

3. Donaldson S, Meza J, Breneman J et al (2001) Results from the IRS-IV randomized trial of hyperfractionated radiotherapy in children with rhabdomyosarcoma-a report from the IRSG. Int J Rad Onc Biol Phys 51:718-28

4. Regine W, Fontanesi J, Kumar P et al (1995) Local tumor control in rhabdomyosarcoma following low-dose irradiation: comparison of group II and select group III patients. Int J Rad Onc Biol Phys 31:485-491

5. Michalski J, Meza J, Breneman J et al (2004) Influence of radiation therapy parameters on outcome in children treated with radiation therapy for localized parameningeal rhabdomyosarcoma in intergroup rhabdomyosarcoma study group trials II-IV. Int J Rad Onc Biol Phys 59:1027-38

6. McDonald MW, Esiashvili N, George BA et al (2008) Intensity modulated radiotherapy with the use of cone-down boost for pediatric head-and-neck rhabdomyosarcoma. Int J Radiati Oncol Biol Phys 72:884-91

7. Douglas JG, Arndt CA, Hawkins DS (2006) Delayed radiotherapy following dose intensive chemotherapy for parameningeal rhabdomyosarcoma (PM-RMS) of childhood. Europ J Cancer 43:1045-1050

8. Smith SC, Lindsley SK, Felgenhauer J (2002) Intensive induction chemotherapy and delayed irradiation in the management of parameningeal rhabdomyosarcoma. J Pediatr Hematol Oncol 25:774-779

9. Lawrence W, Gehan EA, Hays DM et al (1987) Prognostic significance of staging factors of the UICC staging system in childhood rhabdomyosarcoma: a report from the Intergroup Rhabdomyosarcoma Study (IRS-II). J Clin Oncol 5:46-54

10. Cox JD et al (1995) Toxicity criteria of the Radiation Therapy Oncology Group (RTOG) and the European Organization for Research and Treatment of Cancer (EORTC). Int J Radiat Oncol Biol Phys 31:1341-1346

11. Crist WM, Anderson JR, Meza JL et al (2001) Intergroup study IV: results for patients with non-metastatic disease. JCO 19:3091-3102

12. Raney RB, Maurer HM, Anderson JR et al (2001) The Intergroup Rhabdomyosarcoma Study Group (IRSG): major lessons from IRS-I through IRS-IV studies as background for the current IRS$\mathrm{V}$ treatment protocols. Sarcoma 5:9-15 